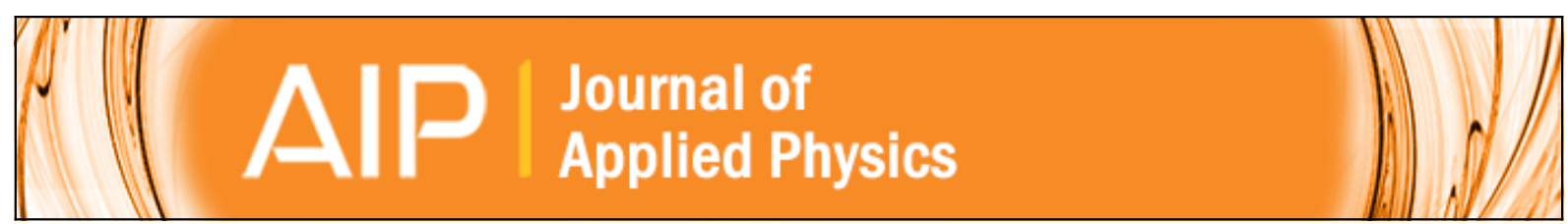

\title{
Electrical detection of magnetic domain wall in Fe4N nanostrip by negative anisotropic magnetoresistance effect
}

Toshiki Gushi, Keita Ito, Soma Higashikozono, Fumiya Takata, Hirotaka Oosato, Yoshimasa Sugimoto, Kaoru

Toko, Syuta Honda, and Takashi Suemasu

Citation: Journal of Applied Physics 120, 113903 (2016); doi: 10.1063/1.4962721

View online: http://dx.doi.org/10.1063/1.4962721

View Table of Contents: http://scitation.aip.org/content/aip/journal/jap/120/11?ver=pdfcov

Published by the AIP Publishing

\section{Articles you may be interested in}

Current-induced three-dimensional domain wall propagation in cylindrical NiFe nanowires

J. Appl. Phys. 119, 153902 (2016); 10.1063/1.4946753

Magnetoresistance effects and spin-valve like behavior of an arrangement of two MnAs nanoclusters

Appl. Phys. Lett. 106, 032401 (2015); 10.1063/1.4906036

Crystalline anisotropic magnetoresistance with two-fold and eight-fold symmetry in (In,Fe)As ferromagnetic semiconductor

Appl. Phys. Lett. 100, 262409 (2012); 10.1063/1.4730955

Magnetoresistance in a lithography defined single constrained domain wall spin-valve Appl. Phys. Lett. 97, 262501 (2010); 10.1063/1.3531666

Resistance of domain walls created by means of a magnetic force microscope in transversally magnetized epitaxial Fe wires

Appl. Phys. Lett. 95, 032504 (2009); 10.1063/1.3187219

The new SR865 2 MHz Lock-In Amplifier ... \$7950

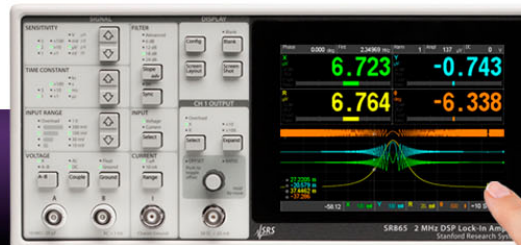

NRS Stanford Research Systems

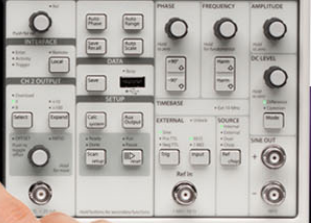

(॰)
Intuitive front-panel operation

Touchscreen data display

Save data \& screen shots to USB flash drive

Embedded web server and iOS app

. Synch multiple SR865s via $10 \mathrm{MHz}$ timebase I/O

View results on a TV or monitor (HDMl output)

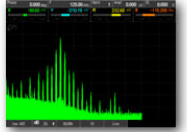

FFT displays

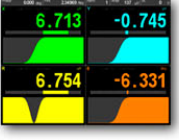

Trend analysis 


\title{
Electrical detection of magnetic domain wall in $\mathrm{Fe}_{4} \mathrm{~N}$ nanostrip by negative anisotropic magnetoresistance effect
}

\author{
Toshiki Gushi, ${ }^{1}$ Keita Ito, ${ }^{1,2,3, a)}$ Soma Higashikozono, ${ }^{1}$ Fumiya Takata, ${ }^{1}$ Hirotaka Oosato, ${ }^{4}$ \\ Yoshimasa Sugimoto, ${ }^{4}$ Kaoru Toko, ${ }^{1}$ Syuta Honda, ${ }^{5}$ and Takashi Suemasu $\left.{ }^{1, b}\right)$ \\ ${ }^{1}$ Institute of Applied Physics, Graduate School of Pure and Applied Sciences, University of Tsukuba, \\ Tsukuba, Ibaraki 305-8573, Japan \\ ${ }^{2}$ Department of Electronic Engineering, Graduate School of Engineering, Tohoku University, \\ Sendai 980-8579, Japan \\ ${ }^{3}$ Japan Society for the Promotion of Science (JSPS), Chiyoda, Tokyo 102-0083, Japan \\ ${ }^{4}$ NIMS Nanofabrication Platform, National Institute for Materials Science, Tsukuba, Ibaraki 305-0047, Japan \\ ${ }^{5}$ Department of Pure and Applied Physics, Faculty of Engineering Science, Kansai University, Suita, \\ Osaka 564-8680, Japan
}

(Received 4 June 2016; accepted 1 September 2016; published online 20 September 2016)

\begin{abstract}
The magnetic structure of the domain wall (DW) of a 30-nm-thick $\mathrm{Fe}_{4} \mathrm{~N}$ epitaxial film with a negative spin polarization of the electrical conductivity is observed by magnetic force microscopy and is well explained by micromagnetic simulation. The $\mathrm{Fe}_{4} \mathrm{~N}$ film is grown by molecular beam epitaxy on a $\mathrm{SrTiO}_{3}(001)$ substrate and processed into arc-shaped ferromagnetic nanostrips $0.3 \mu \mathrm{m}$ wide by electron beam lithography and reactive ion etching with $\mathrm{Cl}_{2}$ and $\mathrm{BCl}_{3}$ plasma. Two electrodes mounted approximately $12 \mu \mathrm{m}$ apart on the nanostrip register an electrical resistance at $8 \mathrm{~K}$. By changing the direction of an external magnetic field $(0.2 \mathrm{~T})$, the presence or absence of a DW positioned in the nanostrip between the two electrodes can be controlled. The resistance is increased by approximately $0.5 \Omega$ when the DW is located between the electrodes, which signifies the negative anisotropic magnetoresistance effect of $\mathrm{Fe}_{4} \mathrm{~N}$. The electrical detection of the resistance change is an important step toward the electrical detection of current-induced DW motion in $\mathrm{Fe}_{4} \mathrm{~N}$. Published by AIP Publishing. [http://dx.doi.org/10.1063/1.4962721]
\end{abstract}

\section{INTRODUCTION}

The application of current-induced magnetic domain wall motion (CIDM) for novel spintronics has been extensively studied both theoretically ${ }^{1-4}$ and experimentally. ${ }^{5-8}$ The CIDM phenomenon is caused by spin transfer torque, which is the transfer of spin angular momentum from conductive electrons to localized electrons. In this way, the magnetic domain wall (DW) can be transferred in the same direction as the electron flow when the current flows in ferromagnetic materials. For balance of the spin angular momentum between the conductive electrons and the localized electrons, ${ }^{9}$ the velocity of the $\mathrm{DW}\left(\boldsymbol{v}_{\mathrm{DW}}\right)$ is given by

$$
v_{\mathrm{DW}}=\frac{P_{\sigma} g \mu_{\mathrm{B}}}{2 e M_{\mathrm{S}}} \boldsymbol{J}
$$

where $P_{\sigma}$ is the spin polarization $\left[P_{\sigma}=\left(\sigma_{\uparrow}-\sigma_{\downarrow}\right) /\left(\sigma_{\uparrow}+\sigma_{\downarrow}\right)\right]$ of the electrical conductivity $(\sigma), g$ is the Lande $g$-factor, $\mu_{\mathrm{B}}$ is the Bohr magneton, $e$ is the elementary charge, $M_{\mathrm{S}}$ is the spontaneous magnetization, and $\boldsymbol{J}$ is the electron flow density. Equation (1) suggests that the DWs move in the same (opposite) direction as the conductive electrons for positive (negative) $P_{\sigma}{ }^{10}$ but no experimental result has yet shown the direction of the DW motion in a material with negative $P_{\sigma}$.

Ferromagnetic $\mathrm{Fe}_{4} \mathrm{~N}$, composed of earth-abundant and nontoxic elements, possesses an anti-perovskite structure

\footnotetext{
${ }^{a)}$ Electronic mail: keita.ito.729@gmail.com

${ }^{\mathrm{b})}$ Electronic mail: suemasu@bk.tsukuba.ac.jp
}

and a Curie temperature $\left(T_{\mathrm{C}}=767 \mathrm{~K}\right)$ that is well above room temperature (RT). ${ }^{11}$ In addition, the $P_{\sigma}$ of $\mathrm{Fe}_{4} \mathrm{~N}$ is theoretically expected to be $-1.0{ }^{12}$ The negative sign of the $P_{\sigma}$ has been deduced from the negative anisotropic magnetoresistance (AMR) ratio, ${ }^{13-15}$ and from the negative spin polarization of the density of states $(D)$ at the Fermi level $\left(E_{\mathrm{F}}\right), P_{\mathrm{D}}$. This spin polarization of the density of states is given by $P_{\mathrm{D}}=\left[D_{\uparrow}\left(E_{\mathrm{F}}\right)-D_{\downarrow}\left(E_{\mathrm{F}}\right)\right] /\left[D_{\uparrow}\left(E_{\mathrm{F}}\right)+D_{\downarrow}\left(E_{\mathrm{F}}\right)\right]<0,{ }^{16,17}$ and the relation that the AMR ratio is proportional to $-P_{\sigma} P_{\mathrm{D}}$ is used. ${ }^{18}$ The AMR ratio is given by

$$
\mathrm{AMR}=\frac{\rho_{/ /}-\rho_{\perp}}{\rho_{\perp}}
$$

where $\rho_{\perp}\left(\rho_{/ /}\right)$is the resistivity when the magnetization is perpendicular (parallel) to the current. Several reports have presented the AMR ratio of $\mathrm{Fe}_{4} \mathrm{~N}$, reporting ratios of $-2.7 \%$ at $5 \mathrm{~K},{ }^{13}-4.3 \%$ at $4.2 \mathrm{~K},{ }^{14}$ and $-6.2 \%$ at $4 \mathrm{~K} .{ }^{15}$ The negative sign of the $P_{\mathrm{D}}$ has been anticipated by theory ${ }^{16}$ and has been confirmed by experiments using spin-resolved photoelectron spectroscopy. ${ }^{17}$ Another report on the inverse tunnel magnetoresistance effect in the $\mathrm{Fe}_{4} \mathrm{~N} / \mathrm{MgO} / \mathrm{CoFeB}$ magnetic tunnel junction structure has also suggested that a negative $P_{\mathrm{D}}$ is present in $\mathrm{Fe}_{4} \mathrm{~N}^{19}$

For ferromagnetic thin films with perpendicular magnetic anisotropy (PMA), CIDM can be detected by various methods such as direct observation using the magnetooptical Kerr effect ${ }^{20-22}$ and electrical measurement using the anomalous Hall effect. ${ }^{23-26}$ For materials with in-plane magnetic anisotropy (IMA), on the other hand, the DW motion is 
detected using the magneto-optical Kerr effect, ${ }^{5,7}$ magnetic force microscopy (MFM) ${ }^{6,8}$ in combination with the change in electrical resistance $(\Delta R)$ owing to the AMR effect, ${ }^{27-30}$ Lorentz MR, and/or DW scattering. ${ }^{31,32}$ In addition, the electrical detection of CIDM using magnon-induced magnetoresistance has been reported in permalloy and $\mathrm{FePt}$ nanostrips. ${ }^{33,34}$ This method is available not only for IMA materials but also for PMA materials, enabling determination of the DW position from the slope of the resistivity versus magnetic flux density minor loop. The $\mathrm{Fe}_{4} \mathrm{~N}$ material exhibits IMA,${ }^{35}$ but there has been no report on the electrical detection of DWs in $\mathrm{Fe}_{4} \mathrm{~N}$ nanostrips, which is of essential importance to detect CIDM in $\mathrm{Fe}_{4} \mathrm{~N}$. This work detects the $\Delta R$ of ferromagnetic $\mathrm{Fe}_{4} \mathrm{~N}$ nanostrips depending upon the presence or absence of a DW between two electrodes mounted on the nanostrips. Electrons scatter in the DW differently from those in the domains adjacent to the DW because the direction of magnetization is different between them.

In this paper, a 30 -nm-thick $\mathrm{Fe}_{4} \mathrm{~N}$ epitaxial thin film was grown on a $\mathrm{SrTiO}_{3}(001)$ substrate (STO) by molecular beam epitaxy (MBE). This film was fabricated into a $0.3-\mu \mathrm{m}$-wide $\mathrm{Fe}_{4} \mathrm{~N}$ nanostrip by electron-beam (EB) lithography and reactive ion etching (RIE) with $\mathrm{Cl}_{2}$ and $\mathrm{BCl}_{3}$ plasma. Finally, the $\mathrm{Fe}_{4} \mathrm{~N}$ nanostrip was used to detect the $\Delta R$ at $8 \mathrm{~K}$ arising from the AMR effect caused by a DW. We also compared the DW magnetic structure obtained by MFM with that calculated using micromagnetic simulation.

\section{METHOD}

\section{A. Formation of $\mathrm{Fe}_{4} \mathrm{~N}$ nanostrips}

We formed a $\mathrm{Ti}(4 \mathrm{~nm}) / \mathrm{Fe}_{4} \mathrm{~N}(30 \mathrm{~nm})$ layered structure on a $\mathrm{SrTiO}_{3}(001)$ substrate at $450{ }^{\circ} \mathrm{C}$ using an ion-pumped MBE system equipped with a high-temperature Knudsen cell for $5 \mathrm{~N}-\mathrm{Fe}$ and with a radio-frequency (RF) $\mathrm{N}_{2}$ plasma. $^{36}$ The epitaxial relationship between $\mathrm{Fe}_{4} \mathrm{~N}$ and STO is known to be $\mathrm{Fe}_{4} \mathrm{~N}[100](001) \| \mathrm{STO}[100](001)$, and the lattice constants of $\mathrm{Fe}_{4} \mathrm{~N}$ and STO are 0.379 and $0.391 \mathrm{~nm}$, respectively. Therefore, the lattice mismatch between $\mathrm{Fe}_{4} \mathrm{~N}$ and STO is $-3 \%$. During growth, we set the Fe deposition rate to $0.95 \mathrm{~nm} / \mathrm{min}$ and the $\mathrm{RF}-\mathrm{N}_{2}$ plasma power to $105 \mathrm{~W}$. To prevent oxidation of the $\mathrm{Fe}_{4} \mathrm{~N}$, the $\mathrm{Fe}_{4} \mathrm{~N}$ was capped with a $\mathrm{Ti}$ layer that may oxidize after air exposure. The electrical resistance $(R)$ of the resulting Ti-O layer is sufficiency large to make any current through this layer negligible. The crystalline quality of the $\mathrm{Fe}_{4} \mathrm{~N}$ was characterized by reflection highenergy electron diffraction (RHEED) along the STO[100] axis and by x-ray diffraction (XRD, RIGAKU Smart Lab.) with $\mathrm{Cu} \mathrm{K} \alpha$ radiation.

Figure 1 shows the fabrication process of the $\mathrm{Fe}_{4} \mathrm{~N}$ nanostrips. First, $0.3-\mu \mathrm{m}$-wide resist [FEP-171/poly(methylglutarimide) (PMGI)] patterns were formed by EB lithography, after which a 50-nm-thick $\mathrm{MgO}$ layer was deposited by EB evaporation. This was followed by a liftoff process to form the mask pattern for etching, and subsequent RIE was carried out with inductively coupled plasma (ICP) of $\mathrm{Cl}_{2}$ $(8 \mathrm{sccm})$ and $\mathrm{BCl}_{3}(2 \mathrm{sccm})$. Any remaining iron chlorides

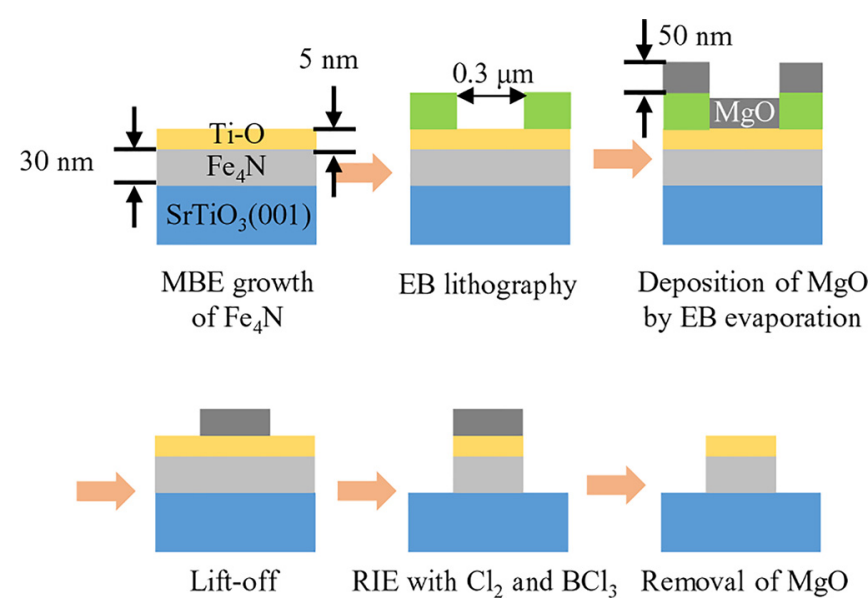

FIG. 1. Fabrication process of $0.3-\mu$ m-wide $\mathrm{Fe}_{4} \mathrm{~N}$ nanostrips. First, bilayer resist [FEP-171/PMGI] patterns were formed by EB lithography, followed by $\mathrm{EB}$ evaporation of the $\mathrm{MgO}$ mask layer. The $0.3-\mu$ m-wide $\mathrm{MaO}$ mask patterns were formed by the liftoff process, and etching of the Ti-O, $\mathrm{Fe}_{4} \mathrm{~N}$, and $\mathrm{SrTiO}_{3}$ was performed by ICP-RIE with $\mathrm{Cl}_{2}$ and $\mathrm{BCl}_{3}$. Finally, the $\mathrm{MgO}$ mask was removed in a diluted $\mathrm{H}_{3} \mathrm{PO}_{4}$ solution.

were then removed by organic solvents, and the $\mathrm{MgO}$ mask was etched in a $\mathrm{H}_{3} \mathrm{PO}_{4}: \mathrm{H}_{2} \mathrm{O}=1: 1000$ solution. Figure 2 presents the geometry of the fabricated nanostrip composed of two perpendicular linear components $0.3 \mu \mathrm{m}$ wide and $7 \mu \mathrm{m}$ long, connected by an $8-\mu \mathrm{m}$ radius arc component (Fig. 2(a)). The linear nanostrip components are aligned along the $\mathrm{Fe}_{4} \mathrm{~N}\langle 110\rangle$ axis, which is the hard axis of the $\mathrm{Fe}_{4} \mathrm{~N}$ thin film. Because of this arrangement, the nanostrip and current (I) are directed nearly parallel to $\mathrm{Fe}_{4} \mathrm{~N}\langle 100\rangle$ around the middle of the arc where, as described later, a DW is formed. It has been reported that the magnitude of the AMR ratio of $\mathrm{Fe}_{4} \mathrm{~N}$ varies depending upon the direction of the current with respect to the crystal orientation, i.e., $-6.2 \%$ with $I \| \mathrm{Fe}_{4} \mathrm{~N}[100]$ and $-1.2 \%$ with $I \| \mathrm{Fe}_{4} \mathrm{~N}[110] .{ }^{15}$ In the arrangement of the nanostrip in this work, the linear components are along the $\mathrm{Fe}_{4} \mathrm{~N}\langle 110\rangle$ and the current flows in a direction nearly parallel to $\mathrm{Fe}_{4} \mathrm{~N}[100]$ around the DW in the arc. Therefore, this nanostrip configuration causes the measured $\Delta R$ value to be larger than those expected when the linear components are aligned along the $\mathrm{Fe}_{4} \mathrm{~N}\langle 100\rangle$ axis. For the resistance measurement, two metal pads made of $\mathrm{Au}(300 \mathrm{~nm}) / \mathrm{Ti}(20 \mathrm{~nm})$ stacks were placed approximately $12 \mu \mathrm{m}$ apart on both ends of the arc by EB evaporation and a liftoff process. The physical morphology of the nanostrip was observed by atomic force microscopy (AFM, HITACHI E-sweep).

\section{B. Domain wall observation and computational details}

The DW was formed in the arc in the following way. The magnetization in the nanostrip aligned along the external magnetic field $(H)$, which was applied in one of the two directions shown by arrows in Figs. 2(b) and 2(d). When the $H$ was then turned off, a DW was either created in the arc (Fig. 2(c)) or not (Fig. 2(e)), depending upon the initial direction of $H$. The magnitude of $H$ was approximately $0.2 \mathrm{~T}$, which was sufficiently large to saturate the 

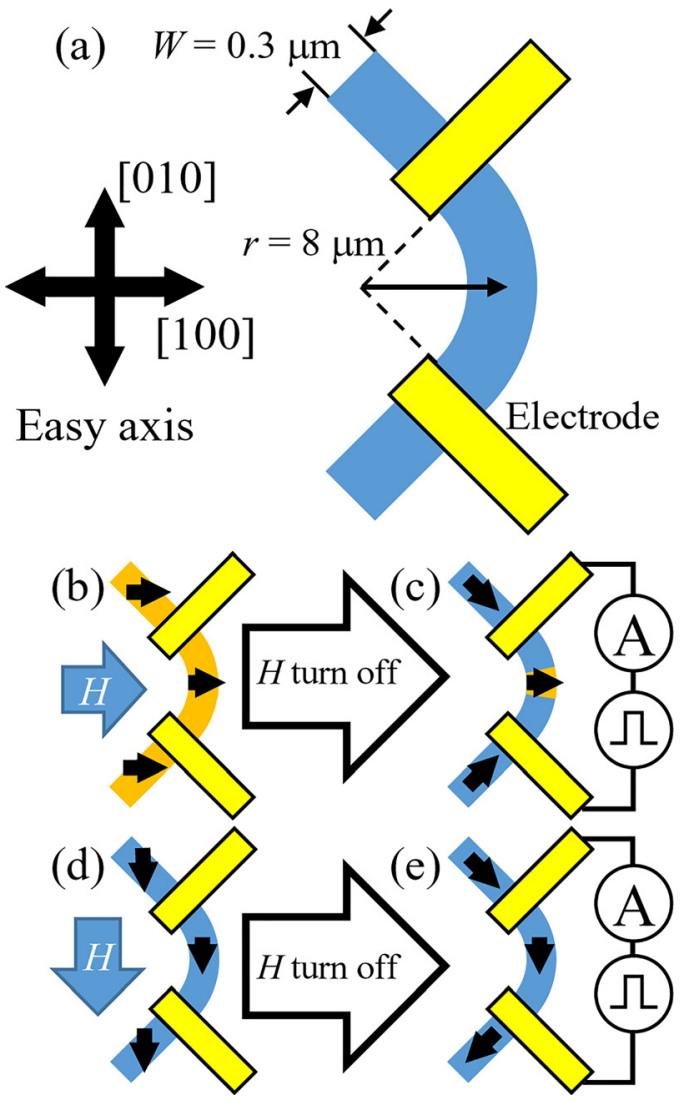

FIG. 2. (a) Schematic drawing of a fabricated nanostrip. The linear components are along the hard axes, $\mathrm{Fe}_{4} \mathrm{~N}\langle 110\rangle$. The radius of the arc component connecting the linear components is $8 \mu \mathrm{m}$ and the width is $0.3 \mu \mathrm{m}$. Two Au/ Ti electrodes are placed on both ends of the arc. (b)-(e) Schematics of resistance measurement cycle, where $R$ was measured 50 times between the two electrodes (yellow rectangles) by applying pulse voltages in (c) and (e). First, (b) $H$ was applied along the $\mathrm{Fe}_{4} \mathrm{~N}[100]$ axis, and (c) $R$ was measured after $H$ was turned off. In (d), $H$ was applied along the $\mathrm{Fe}_{4} \mathrm{~N}[010]$ axis to remove the DW from the nanostrip, and (e) $R$ was measured after $H$ was turned off. The pulse voltage has a $50 \mathrm{~ms}$ width, a $10 \mathrm{~s}$ period, and a $0.20 \mathrm{~V}$ height.

magnetization in a soft magnetic material like $\mathrm{Fe}_{4} \mathrm{~N}$. The DW in the $\mathrm{Fe}_{4} \mathrm{~N}$ nanostrip was observed by MFM (HITACHI E-sweep) at RT.

The magnetic domain structure in the $\mathrm{Fe}_{4} \mathrm{~N}$ nanostrip after turning $H$ off was calculated by micromagnetic simulation using the Object Oriented MicroMagnetic Framework (OOMMF) ${ }^{37}$ The material parameters used for the simulations were the magnetization $M_{\mathrm{S}}=1.43 \mathrm{MA} / \mathrm{m}$, the crystalline magnetic anisotropy constant $K_{\mathrm{C}}=3.0 \times 10^{4} \mathrm{~J} / \mathrm{m}^{3}$, and the exchange stiffness $A=15 \mathrm{pJ} / \mathrm{m}$. Among these, the $M_{\mathrm{S}}$ and $K_{\mathrm{C}}$ were measured values, ${ }^{35}$ while the value of $A$ for $\mathrm{Fe}_{4} \mathrm{~N}$ was approximated from that for $\mathrm{Fe}$ using the relationship that $A$ is nearly proportional to $T_{\mathrm{C}} \cdot{ }^{38}$ The damping constant was set to $\alpha=1$ to accelerate the calculation with the expectation that the value of $\alpha$ has no effect on equilibrium states. The cell size used for the simulation was $5 \times 5 \times 5 \mathrm{~nm}^{3}$, and the same geometry was used as that shown in Fig. 2(a). As the initial state, the magnetization was set homogeneously along $H$ and then relaxed depending upon the crystalline and shape anisotropy. For an indication of the MFM contrast, the volume density of the magnetic charge $-\operatorname{div} \boldsymbol{M}$ was calculated for each cell.

\section{Electrical detection of DW}

The resistance measurements were carried out at $8 \mathrm{~K}$, where the cooling was accomplished by a closed-cycle $\mathrm{He}$ cryostat to enhance the AMR ratio. We first measured pulse currents by applying pulse voltages between the electrodes in the state shown in Fig. 2(c), and then converted them to $R$ values. The pulse width, period, and height were $50 \mathrm{~ms}$, $1.0 \mathrm{~s}$, and $0.20 \mathrm{~V}$, respectively, and each measurement was repeated 50 times. Next, $H$ was applied as shown in Fig. 2(d) to remove the DW from the nanostrip, and the $R$ value was again measured in the state shown in Fig. 2(e).

\section{RESULTS AND DISCUSSION}

\section{A. $\mathrm{Fe}_{4} \mathrm{~N}$ nanostrips}

Figure 3 presents the $\omega-2 \theta$ XRD and RHEED patterns of the as-grown $\mathrm{Fe}_{4} \mathrm{~N}$ film. The streaky RHEED pattern and the presence of XRD peaks from only $c$-axis-oriented $\mathrm{Fe}_{4} \mathrm{~N}$ such as (001), (002), and (004) demonstrate the epitaxial growth of the $\mathrm{Fe}_{4} \mathrm{~N}$ film. In addition, superlattice reflections in the RHEED pattern together with the (001) peak in the XRD pattern clearly indicate that the $\mathrm{N}$ atom exists at the body center of the face-centered cubic lattice. Figure 4 shows the AFM top view of the fabricated nanostrip and its cross-sectional profile, showing the formation of the $\mathrm{Fe}_{4} \mathrm{~N}$ film into uniform nanostrips with smooth surfaces.

\section{B. Domain wall simulation and experiment}

Figures 5(a) and 5(b) present the MFM images of the $\mathrm{Fe}_{4} \mathrm{~N}$ nanostrip captured during the states illustrated in Figs. 2(e) and 2(c), respectively. It can be seen that we successfully created (erased) a vortex DW around the middle of the arc by applying $H$ along the normal (tangent) of the arc. The width of the DW is approximately $0.8 \mu \mathrm{m}$, as shown in Fig. 5(b). Figure 5(c) shows the result of simulation, which also suggests the formation of a vortex DW. The measured magnetic structure of the DW in Fig. 5(b) is nicely described by the simulation.

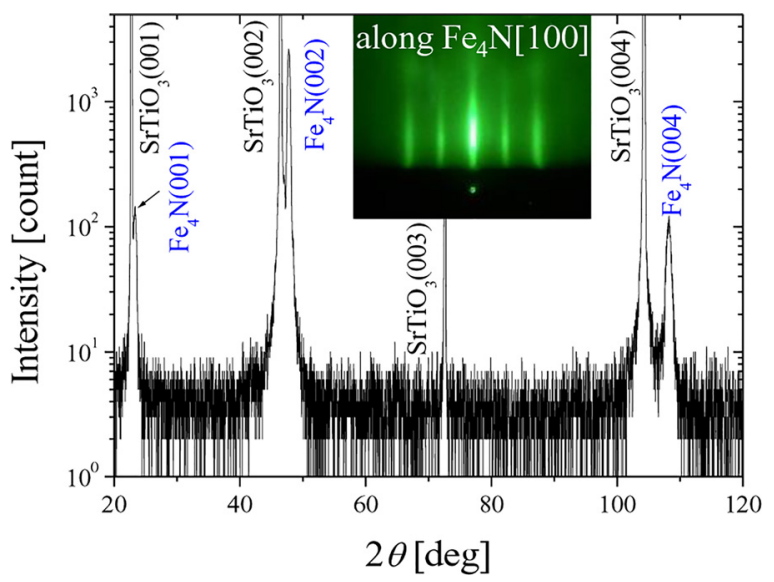

FIG. 3. $\omega-2 \theta$ XRD pattern of the $\mathrm{Fe}_{4} \mathrm{~N}$ epitaxial film. (Inset) RHEED pattern observed along the $\mathrm{Fe}_{4} \mathrm{~N}[100]$ axis. 

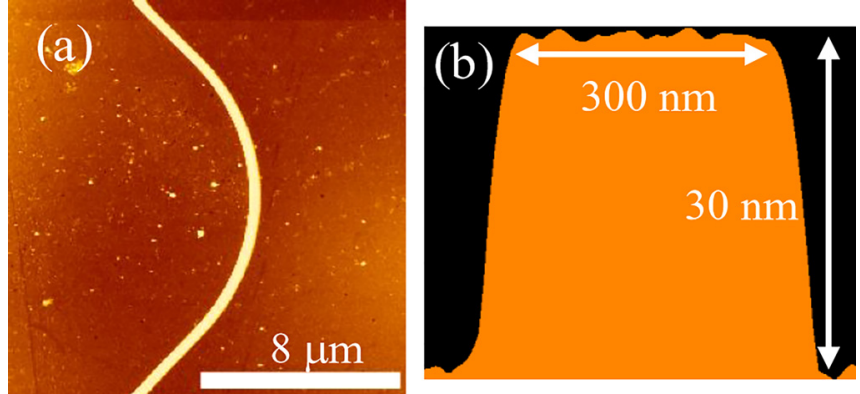

FIG. 4. (a) AFM topographic image and (b) cross-sectional profile of the nanostrip.

\section{Resistance change}

Figures 6(a) and 6(b) show the resistance change $\Delta R$ of the $\mathrm{Fe}_{4} \mathrm{~N}$ nanostrip with and without a DW present in the arc, measured at $8 \mathrm{~K}$. In the initial state shown in Fig. 2(e), i.e., no DW present in the arc, $R=291.6 \Omega$. When a DW is introduced in the arc and the state of magnetization is changed from the conditions in Fig. 2(e) to that in Fig. 2(c), $R$ increases by approximately $0.5 \Omega$, as shown in Fig. 6(a). We next changed the state of magnetization from that in Fig. 2(c) to that shown in Fig. 2(e), effectively removing the DW from the arc, and found that $R$ decreases by approximately $0.5 \Omega$. These measured $\Delta R$ values are reliable from the viewpoint of the signal-to-noise ratio, and similar results were obtained with excellent repeatability. These results demonstrate that the presence of a DW in the arc of the $\mathrm{Fe}_{4} \mathrm{~N}$ nanostrip increases the nanostrip $R$, which we can attribute to the negative AMR effect caused by the negative $P_{\sigma}$ of $\mathrm{Fe}_{4} \mathrm{~N}{ }^{13-15}$ Herein, we attempt to estimate the magnitude of $\Delta R$ arising
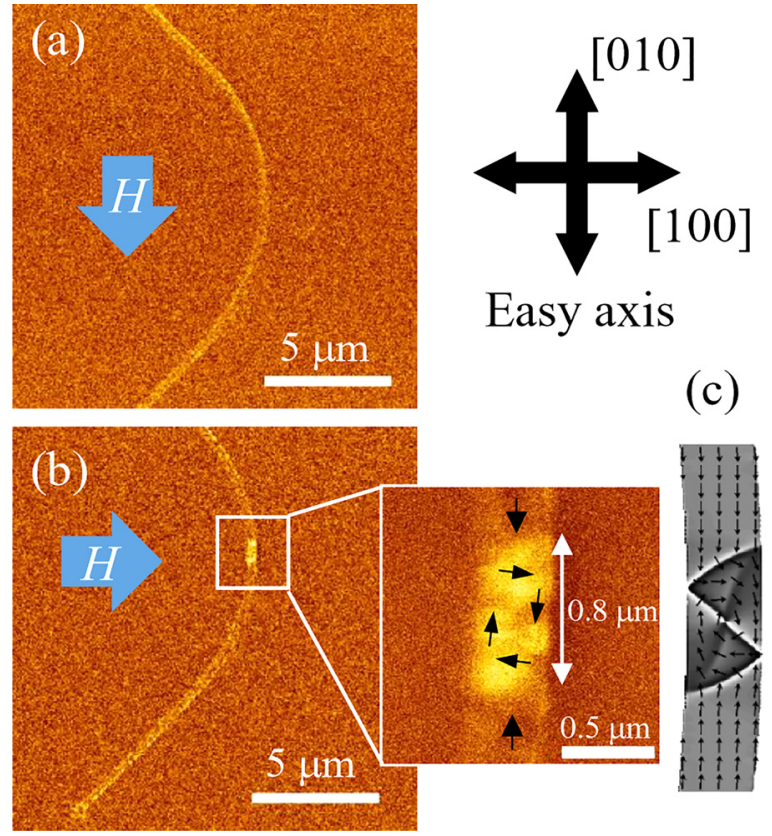

FIG. 5. (a)(b) MFM images captured at RT after $H(0.2 \mathrm{~T})$ was applied along the arrows and then turned off. The DW is absent in the arc in (a) but present in (b). The magnified view in (b) shows the existence of a vortex DW. (c) Simulation result by $\mathrm{OOMMF}^{37}$ using the same dimensions of the nanostrip and the material parameters $M_{\mathrm{S}}=1.43 \mathrm{MA} / \mathrm{m}, K_{\mathrm{C}}=3.0 \times 10^{4} \mathrm{~J} / \mathrm{m}^{3}, A=15$ $\mathrm{pJ} / \mathrm{m}$, and $\alpha=1$.

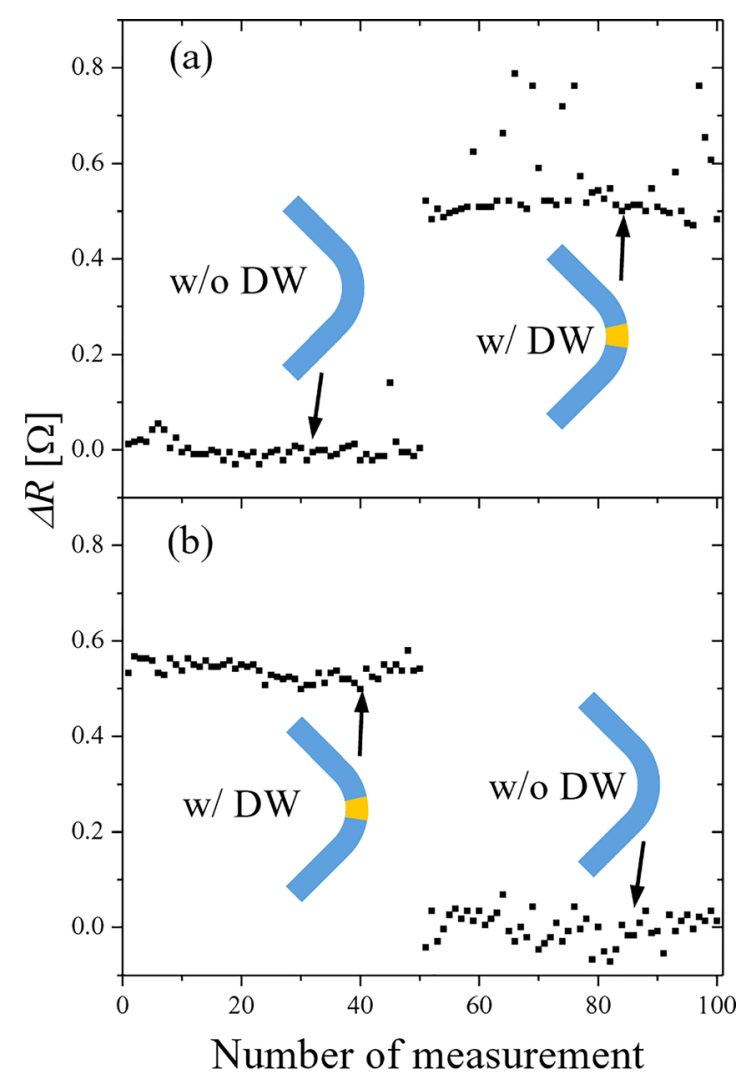

FIG. 6. Resistance change defined by $\Delta R=R_{\mathrm{w} /}-R_{\mathrm{w} / \mathrm{o}}$ of the $\mathrm{Fe}_{4} \mathrm{~N}$ nanostrip obtained (a) when the DW is absent and then introduced into the arc and (b) when the DW is present and then removed from the arc. $R_{\mathrm{w} /}$ and $R_{\mathrm{w} / \mathrm{o}}$ are the resistances measured with or without the DW, respectively. $\Delta R$ is approximately $0.5 \Omega$.

from the AMR effect, although other factors such as the Lorentz MR exist. The Lorentz MR ratio is proportional to $\left(B_{\perp} / \rho\right)^{2}$, where $B_{\perp}$ is the component of the magnetic flux density $B(=H+4 \pi M)$ perpendicular to $I$ and $\rho$ is the resistivity. Here, $M$ is the magnetization. Therefore, the Lorentz MR is small in materials with high resistivity such as $\mathrm{Fe}_{4} \mathrm{~N} .{ }^{39}$ In ferromagnetic materials, the resistivity $\rho$ varies depending upon the angle between the current and the magnetization caused by the AMR effect. Major ferromagnetic materials such as NiFe have a positive AMR ratio such that $\rho_{/ /}>\rho_{\perp}$, causing a local variation of the magnetization to exist within the DW. Within this variation, some magnetic moments possess a component perpendicular to the current and thereby contribute to a lower resistance. ${ }^{27-30}$ However, $\mathrm{Fe}_{4} \mathrm{~N}$ exhibits a negative AMR ratio caused by a negative $P_{\sigma}$, such that $\rho_{/ /}-\rho_{\perp}<0$. Therefore, in the state illustrated in Fig. 2(c), the resistivity of the DW in the $\mathrm{Fe}_{4} \mathrm{~N}$ nanostrip $\left(\approx \rho_{\perp}\right)$ becomes larger than that of the other nanostrip areas $\left(\approx \rho_{/ /}\right)$. Assuming that the DW has a rectangular parallelepiped shape with height $t$, width $W$, and length $\Delta x$ along the arc, and that the magnetization is perpendicular to the longitudinal direction in the DW as shown in Fig. 2(c), $\Delta R$ is given by

$$
\Delta R=R_{\mathrm{W} /}-R_{\mathrm{W} / \mathrm{O}} \cong \frac{\Delta x}{W t}\left(\rho_{\perp}-\rho_{/ /}\right)=-\mathrm{AMR} \times \frac{\Delta x}{W t} \rho_{\perp} .
$$

Here, $R_{\mathrm{w} /}$ and $R_{\mathrm{w} / \mathrm{o}}$ are the resistances of the nanostrip measured with or without the $\mathrm{DW}$, respectively. The $R_{\mathrm{w} /}$ and $R_{\mathrm{w} / \mathrm{o}}$ are 
approximately given by $R_{\mathrm{W} /} \cong\left[\rho_{\perp} \Delta x+\rho_{/ /}(l-\Delta x)\right] /(W t)$ and $R_{\mathrm{W} / \mathrm{O}} \cong\left(\rho_{/ /} l\right) /(W t)$, respectively, where $l$ is the length of the nanostrip. In this experiment, $\Delta x$ is approximately $0.8 \mu \mathrm{m}$ and the width and height of the nanostrip are $0.3 \mu \mathrm{m}$ and $30 \mathrm{~nm}$, respectively. Because $\rho_{\perp}$ is about $34 \mu \Omega \cdot \mathrm{cm}$ at $8 \mathrm{~K}$ for $\mathrm{Fe}_{4} \mathrm{~N},{ }^{13}$ $\Delta R$ is derived to be approximately $0.8 \Omega$ using Eq. (3). In the case of a vortex DW, which is true for this work, some magnetic moments in the DW have a component parallel to the current and thereby contribute to a lower resistance. Therefore, we anticipate $\Delta R$ to be smaller than the $0.8 \Omega$ calculated using the rectangular-parallelepiped-shaped DW model. In actuality, the measured value of $\Delta R$ shown in Fig. 6 is approximately $0.5 \Omega$, which is slightly smaller than that estimated from Eq. (3). We thereby conclude that the measured $\Delta R$ is well explained by the AMR effect taking place in the DW. On the basis of these results, the CIDM of the $\mathrm{Fe}_{4} \mathrm{~N}$ nanostrip can be detected electrically owing to a sufficiently large resistance increase.

\section{CONCLUSION}

A 30-nm-thick $\mathrm{Fe}_{4} \mathrm{~N}$ epitaxial film was grown and processed into $0.3-\mu \mathrm{m}$-wide arc-shaped nanostrips by EB lithography and ICP RIE using $\mathrm{Cl}_{2}$ and $\mathrm{BCl}_{3}$ plasmas. By changing the direction of $H$, the presence or absence of a DW could be controlled in the $\mathrm{Fe}_{4} \mathrm{~N}$ nanostrip. Furthermore, micromagnetic simulations using OOMMF explained the formation of a vortex DW. When the DW was introduced in the arc between the two electrodes, the $R$ value measured at $8 \mathrm{~K}$ was increased by approximately $0.5 \Omega$. On the other hand, the $R$ value was decreased by approximately $0.5 \Omega$ when the DW was removed from the nanostrip. This resistance change was well explained using the resistivity of the DW estimated from the already-reported negative AMR ratio of $\mathrm{Fe}_{4} \mathrm{~N}$ at $8 \mathrm{~K}$ for a simple rectangular parallelepiped shape. This is an important step toward the electrical detection of CIDM in $\mathrm{Fe}_{4} \mathrm{~N}$ nanostrips.

\section{ACKNOWLEDGMENTS}

This work was supported in part by the Japan Society for the Promotion of Science (JSPS) Grants-in-Aid for Scientific Research (A) (No. 26249037) and JSPS Fellows (Nos. 14J01804 and 16J02879), the National Institute for Materials Science Nanofabrication Platform in the "Nanotechnology Platform Project" sponsored by the Ministry of Education, Culture, Sports, Science and Technology (MEXT), and the OPEN FACILITY, Research Facility Center for Science and Technology, University of Tsukuba.

\footnotetext{
${ }^{1}$ L. Berger, Phys. Rev. B 54, 9353 (1996).

${ }^{2}$ J. C. Slonczewski, J. Magn. Magn. Mater. 159, L1 (1996).

${ }^{3}$ G. Tatara and H. Kohno, Phys. Rev. Lett. 92, 086601 (2004).

${ }^{4}$ Z. Li and and S. Zhang, Phys. Rev. Lett. 92, 207203 (2004).

${ }^{5}$ N. Vernier, D. A. Allwood, D. Atkinson, M. D. Cooke, and R. P. Cowburn, Europhys. Lett. 65, 526 (2004).

${ }^{6}$ A. Yamaguchi, T. Ono, S. Nasu, K. Miyake, K. Mibu, and T. Shinjo, Phys. Rev. Lett. 92, 077205 (2004).

${ }^{7}$ G. S. D. Beach, C. Knutson, C. Nistor, M. Tsoi, and J. L. Erskine, Phys. Rev. Lett. 97, 057203 (2006).
}

${ }^{8}$ S. S. P. Parkin, M. Hayashi, and L. Thomas, Science 320, 190 (2008).

${ }^{9}$ A. Thiaville, Y. Nakatani, J. Miltat, and Y. Suzuki, Europhys. Lett. 69, 990 (2005).

${ }^{10}$ T. Gushi, K. Ito, S. Honda, Y. Yasutomi, K. Toko, H. Oosato, Y. Sugimoto, K. Asakawa, N. Ota, and T. Suemasu, Jpn. J. Appl. Phys., Part 1 54, 028003 (2015).

${ }^{11}$ G. Shirane, W. J. Takei, and S. L. Ruby, Phys. Rev. 126, 49 (1962).

${ }^{12}$ S. Kokado, N. Fujima, K. Harigaya, H. Shimizu, and A. Sakuma, Phys. Rev. B 73, 172410 (2006).

${ }^{13}$ K. Ito, K. Kabara, H. Takahashi, T. Sanai, K. Toko, T. Suemasu, and M. Tsunoda, Jpn. J. Appl. Phys., Part 1 51, 068001 (2012).

${ }^{14}$ M. Tsunoda, Y. Komasaki, S. Kokado, S. Isogami, C. C. Chen, and M. Takahashi, Appl. Phys. Express 2, 083001 (2009).

${ }^{15}$ M. Tsunoda, H. Takahashi, S. Kokado, Y. Komasaki, A. Sakuma, and M. Takahashi, Appl. Phys. Express 3, 113003 (2010).

${ }^{16}$ A. Sakuma, J. Magn. Magn. Mater. 102, 127 (1991).

${ }^{17}$ K. Ito, K. Okamoto, K. Harada, T. Sanai, K. Toko, S. Ueda, Y. Imai, T. Okuda, K. Miyamoto, A. Kimura, and T. Suemasu, J. Appl. Phys. 112, 013911 (2012).

${ }^{18}$ S. Kokado, M. Tsunoda, K. Harigaya, and A. Sakuma, J. Phys. Soc. Jpn. 81, 024705 (2012).

${ }^{19}$ Y. Komasaki, M. Tsunoda, S. Isogami, and M. Takahashi, J. Appl. Phys. 105, 07 C928 (2009).

${ }^{20}$ M. Yamanouchi, D. Chiba, F. Matsukura, T. Dietl, and H. Ohno, Phys. Rev. Lett. 96, 096601 (2006).

${ }^{21}$ T. A. Moore, I. M. Miron, G. Gaudin, G. Serret, S. Auffret, B. Rodmacq, A. Schuhl, S. Pizzini, J. Vogel, and M. Bonfim, Appl. Phys. Lett. 93, 262504 (2008).

${ }^{22}$ L. S. E. Alvarez, K.-Y. Wang, S. Lepadatu, S. Landi, S. J. Bending, and C. H. Marrows, Phys. Rev. Lett. 104, 137205 (2010).

${ }^{23}$ H. Tanigawa, T. Koyama, G. Yamada, D. Chiba, S. Kasai, S. Fukami, T. Suzuki, N. Ohshima, N. Ishiwata, Y. Nakatani, and T. Ono, Appl. Phys. Express 2, 053002 (2009).

${ }^{24}$ J. Heinen, O. Boulle, K. Rousseau, G. Malinowski, M. Kläui, H. J. M. Swagten, B. Koopmans, C. Ulysse, and G. Faini, Appl. Phys. Lett. 96, 202510 (2010).

${ }^{25}$ T. Koyama, H. Hata, K. J. Kim, T. Moriyama, H. Tanigawa, T. Suzuki, Y. Nakatani, D. Chiba, and T. Ono, Appl. Phys. Express 6, 033001 (2013).

${ }^{26}$ Z. Meng, M. Kumar, J. Qiu, G. Han, K. L. Teo, and D. T. Ngo, J. Phys. D: Appl. Phys. 47, 345001 (2014).

${ }^{27}$ M. Kläui, C. A. F. Vaz, J. Rothman, J. A. C. Bland, W. Wernsdorfer, G. Faini, and E. Cambril, Phys. Rev. Lett. 90, 097202 (2003).

${ }^{28}$ M. Hayashi, L. Thomas, C. Rettner, R. Moriya, Y. B. Bazaliy, and S. S. P. Parkin, Phys. Rev. Lett. 98, 037204 (2007).

${ }^{29}$ M. Kläui, C. A. F. Vaz, J. A. C. Bland, W. Wernsdorfer, G. Faini, E. Cambril, L. J. Heyderman, F. Nolting, and U. Rüdiger, Phys. Rev. Lett. 94, 106601 (2005).

${ }^{30}$ M. Hayashi, L. Thomas, C. Rettner, R. Moriya, X. Jiang, and S. S. P. Parkin, Phys. Rev. Lett. 97, 207205 (2006).

${ }^{31}$ T. Taniyama, I. Nakatani, T. Namikawa, and Y. Yamazaki, Phys. Rev. Lett. 82, 2780 (1999).

${ }^{32}$ A. D. Kent, J. Yu, U. Rüdiger, and S. S. P. Parkin, J. Phys.: Condens. Matter. 13, R461 (2001).

${ }^{33}$ V. D. Nguyen, C. Naylor, L. Vila, A. Marty, P. Laczkowski, C. Beigne, L. Notin, Z. Ishaque, and J. P. Attané, Appl. Phys. Lett. 99, 262504 (2011).

${ }^{34}$ V. D. Nguyen, L. Vila, P. Laczkowski, A. Marty, T. Faivre, and J. P. Attané, Phys. Rev. Lett. 107, 136605 (2011).

${ }^{35}$ J. L. Costa-Krämer, D. M. Borsa, J. M. García-Martín, M. S. MartínGonzález, D. O. Boerma, and F. Briones, Phys. Rev. B 69, 144402 (2004).

${ }^{36}$ T. Sanai, K. Ito, K. Toko, and T. Suemasu, J. Cryst. Growth 357, 53 (2012).

${ }^{37}$ M. J. Donahue and D. G. Porter, OOMMF user's guide, version 1.0. Technical Report No. NISTIR 6376, National Institute of Standards and Technology, Gaithersburg, MD, 1999. URL http://math.nist.gov/oommf. Version 1.2a of the software was used.

${ }^{38}$ K. Ito, N. Rougemaille, S. Pizzini, S. Honda, N. Ota, T. Suemasu, and O. Fruchart, preprint arXiv:1511.07520 (2015).

${ }^{39}$ K. Ito, K. Kabara, T. Sanai, K. Toko, Y. Imai, M. Tsunoda, and T. Suemasu, J. Appl. Phys. 116, 053912 (2014). 